\title{
Design and Simulation of Anti-Swing Control System Based on Input Shaping Technique \\ WANG Xiaochao
}

Institute of Rail Transit, Tongji University, Shanghai, China

wangxiaochao@tongji.edu.cn

Keywords: Cranes, Anti-swing, Lagrange equations, Input shaping controller, Simulink simulation.

\begin{abstract}
Based on Lagrange equation, the dynamic model of crane system is established, and the transfer function of crane operating system is deduced. This paper presents a crane anti-swing solution, which is to establish the anti-swing control model basis the input shaping technique. Three input shaping controllers are designed and realized in this paper, including zero vibration (ZV), zero vibration and derivation (ZVD) and extra insensitivity (EI) input shaping controller. The crane system with the input shaping controller is simulated by Simulink toolbox of MATLAB software. The simulation results show that the swing of crane system with the input shaping controller is significantly restrained when the load reaches the designated position.
\end{abstract}

\section{基于输入整形技术的防摇控制系统设计与仿真}

\author{
王小超 \\ 同济大学铁道与城市轨道交通研究院, 上海, 中国 \\ wangxiaochao@tongji.edu.cn
}

关键词: 吊车, 防摇摆, 拉格朗日方程, 输入整形器, Simulink仿真

中文摘要. 基于Lagrange方程建立吊车系统动力学模型, 进而推导出吊车运行系统的传递函 数。提出了本文的吊车消摆方案, 即建立基于输入整形技术的防摇控制模型, 设计并实现吊 车系统的零振动、零振动微分和极不灵敏三种输入整形控制器。将输入整形器应用到吊车动 力学系统中, 利用MATLAB软件的Simulink工具箱对增加了输入整形器的吊车动力学系统进 行仿真。仿真结果表明, 加了输入整形器之后的吊车系统, 当负载到达指定位置后摆动情况 得到明显抑制。

\section{1. 引言}

吊车在回转、搬运、制动等过程中，所提升的重物由于自身惯性会出现摆动现象，这会 影响搬运效率, 甚至对地面工作人员产生危险隐患。当货物通过钢丝绳被吊车吊起之后, 如 何能实现货物的平稳运行, 和垂直落地, 已经成为快速提高搬运效率和增强作业安全性急需 要解决的问题。

本文利用Lagrange方程来建立吊车防摇系统的数学模型 ${ }^{[1]}$, 求出吊车系统的传递函数。然 后建立基于输入整形技术的防摇摆模型, 主要研究了零振动、零振动微分和极不灵敏三种输 入整形控制器 ${ }^{[2]}$, 分析了各自的性能和适合的工作环境。最后的模型仿真部分, 利用MATLAB 中Simulink工具箱对增加了输入整形器的吊车系统进行仿真 ${ }^{[3]}$, 仿真结果显示负载摆动情况得 到明显抑制，验证了文章模型的正确性。 


\section{2. 吊车运行系统动力学分析与建模}

吊车系统的简化动力学模型如图 1 所示 ${ }^{[4]}$ :

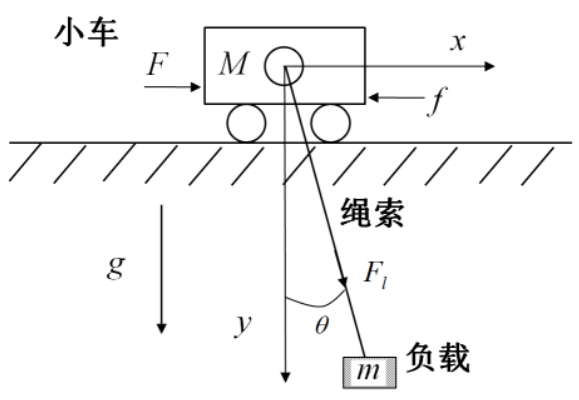

图1 吊车运行系统动力学简化模型

对于整个吊车运行系统, 以力 $F$ 方向为 $x$ 轴正方向, 垂直地面向下为 $y$ 轴正方向, 建立直 角坐标系。分别选择水平位移 $x$ 和摆角 $\theta$ 为系统的广义坐标, 建立具有 2 个广义坐标下的吊车 防摇摆系统模型, $M$ 和 $m$ 分别表示吊车与吊重的质量, $l$ 为绳索长度, $F$ 为吊车水平驱动力, $\theta$ 为重物摆动过程中绳索与坚直方向的夹角, $\mu$ 为吊车与轨道之间的摩擦系数。应用Lagrange 方程得到系统动力学模型。

$$
\left\{\begin{array}{l}
(M+m) \ddot{x}+m l \ddot{\theta}+\mu \dot{x}=F \\
l \ddot{\theta}+\ddot{x}+g \theta=0
\end{array}\right.
$$

对式(1)进行拉普拉斯变换：

$$
\left\{\begin{array}{l}
F(s)=\left(M s^{2}+\mu s\right) X(s)-m g \theta(s) \\
s^{2} X(s)=-\left(l s^{2}+g\right) \theta(s)
\end{array}\right.
$$

整理后得到吊车系统传递函数:

$$
\left\{\begin{array}{l}
G_{1}(s)=\frac{X(s)}{F(s)}=\frac{l s^{2}+g}{M l s^{4}+\mu l s^{3}+(M+m) g s^{2}+\mu g s} \\
G_{2}(s)=\frac{\theta(s)}{F(s)}=\frac{-s^{2}}{M l s^{4}+\mu l s^{3}+(M+m) g s^{2}+\mu g s}
\end{array}\right.
$$

根据相关实验设备, 得到吊车系统输入参数的值, $M=5 \mathrm{~kg}, m=10 \mathrm{~kg}, l=2 \mathrm{~m}, \mathrm{~g}=9.8 \mathrm{~m} / \mathrm{s}^{2}$, $\mu$ 经查表确定为 0.2 , 带入式(3)计算出吊车运行系统的传递函数:

$$
\left\{\begin{array}{l}
G_{1}(s)=\frac{X(s)}{F(s)}=\frac{0.2 s^{2}+0.98}{s^{4}+0.04 s^{3}+14.7 s^{2}+0.196 s} \\
G_{2}(s)=\frac{\theta(s)}{F(s)}=\frac{-0.1 s^{2}}{s^{4}+0.04 s^{3}+14.7 s^{2}+0.196 s}
\end{array}\right.
$$

\section{3. 输入整形技术与吊车系统输入整形器设计}

\section{1 输入整形控制基本原理}

输入整形技术是序列脉冲和系统的一个期望指令卷积得到新的系统输入信号, 该输入信 号可以使系统以无残留振动或低于某一振动水平运动(图2)。在输入整形器作用下二阶系统的 残留振荡百分比如式(5)所示 ${ }^{[5]}$ :

$$
V(\omega, \xi)=e^{-\xi \omega t_{n}} \sqrt{[C(\omega, \xi)]^{2}+[s(\omega, \xi)]^{2}}
$$

其要满足时间优化约束和脉冲幅值约束: 


$$
\min \left(t_{n}\right), \quad \sum_{i=1}^{n} A_{i}=1
$$

其中 $t_{n}$ 为最后一个脉冲的时滞。

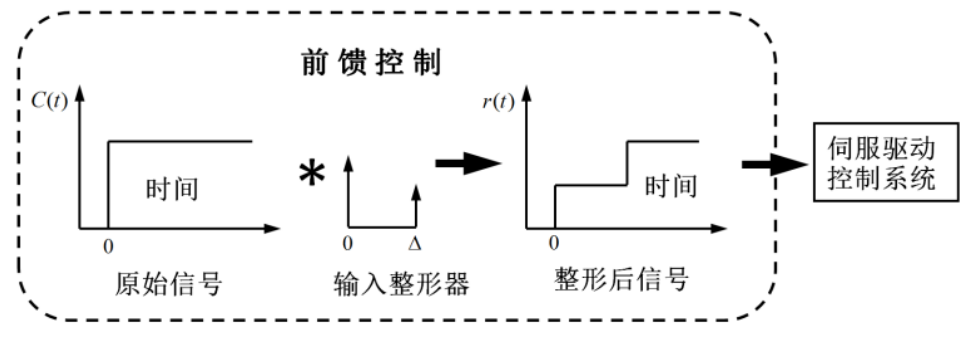

图2 输入整形信号的产生

\section{2 输入整形器的三种基本形式}

(1) 零振动输入整形器 (Zero Vibration, ZV)

当残留振荡百分比 $V(\omega, \xi)=0$ 时, 得到的脉冲序列称之为 ZV 整形器通过零极点对消的输入 整形器设计方法, 可计算得到脉冲幅值和时滞为:

$$
A_{1}=\frac{1}{1+K}, A_{2}=\frac{K}{K+1}, t_{1}=0, t_{2}=T
$$

其中 $T$ 为系统的半振动周期, $T=\pi /\left(\omega_{n} \sqrt{1-\xi^{2}}\right), \quad K=e^{-\xi \pi / \sqrt{1-\xi^{2}}}$ 。

(2) 零振动微分输入整形器 (Zero Vibration and Derivation,ZZVD)

为了提高整形器的鲁棒性, 可以对残留振动式(5)及其微分方程进行约束, 求解鲁棒性更 好的输入整形器, 即ZVD输入整形器。通过相应计算, 可得到其脉冲幅值和时滞:

$$
A_{1}=\frac{1}{1+2 K+K^{2}}, A_{2}=\frac{2 K}{1+2 K+K^{2}}, A_{3}=\frac{K^{2}}{1+2 K+K^{2}}, t_{1}=0, t_{2}=T, t_{3}=2 T
$$

其中 $T$ 为系统的半振动周期, $T=\pi /\left(\omega_{n} \sqrt{1-\xi^{2}}\right), K=e^{-\xi \pi / \sqrt{1-\xi^{2}}}$ 。

(3) 极不灵敏输入整形器(Extra Insensitivity,EI)

ZV输入整形器和ZVD输入整形器都可以完全消除系统残留震荡, 如果不要求系统残留振 动为零, 而是保持在相对较小的非零值 $V_{t o l}$, 但在比无阻尼固有频率稍高的频率 $\omega_{h}$ 和稍低的频 率 $\omega_{l}$ 处的残留震荡为零, 在无阻尼固有频率 $\omega_{n}$ 处残留振荡幅值对 $\omega_{n}$ 的微分为零, 这样输入整 形器就可以确保频率在区间 $\left[\omega_{l}, \omega_{h}\right]$ 内使系统残留震荡小于或等于允许残留震荡幅值 $V_{t o l}$, 据此 设计的输入整形器为EI输入整形器。根据整形器的约束, 得到以下约束方程:

$$
V(\omega, \xi)=V_{t o l}, V\left(\omega_{l}, \xi\right)=0, V\left(\omega_{h}, \xi\right)=0
$$

由此计算得到EI输入整形器的脉冲幅值和时滞:

$$
A_{1}=A_{3}, A_{2}=1-2 A_{1}, t_{1}=0, t_{2}=T, t_{3}=2 T
$$

其中 $A_{1}=A_{3}=\left(1+V_{t o l}\right) / 4, A_{2}=\left(1-V_{t o l}\right) / 2, T=\pi /\left(\omega_{n} \sqrt{1-\xi^{2}}\right)$

总结来说, 在工况较好, 即系统输入参数一定, 并且不受外部影响的情况下, 使用ZV整 形器较为合理, 它能够完全消除摆动, 并且用时最少。当系统输入参数相对不确定或者存在 较小外部影响的时候, 应该使用ZVD整形器, 它也可以达到负载的完全消摆, 只是消摆时间 相对较长。当工况环境很差, 输入参数不确定, 并且受较大外部环境影响时, 可以考虑使用 $\mathrm{EI}$ 整形器。虽然EI不能完全消除负载的摆动, 但它能够控制负载的摆动处于一个很小的范围 内。虽然EI整形器的时滞与ZVD整形器相同, 但是它的鲁棒性比ZVD强了很多, 因此, 同样 的时滞条件下, 优先选择EI整形器。 


\section{3 吊车系统输入整形器设计}

阻尼在系统中主要作用是可以抑制负载摆动, 由于吊车系统阻尼较小, 可以假设模型为 非阻尼系统。施加外力时, 系统将按照自振频率持续振荡, 根据系统输入参数 $M=5 \mathrm{~kg}$, $m=10 \mathrm{~kg}, l=2 m, g=9.8 \mathrm{~m} / \mathrm{s}^{2}$, 得到系统自振频率:

$$
\omega_{n}=\sqrt{\frac{g}{l}\left(1+\frac{m}{M}\right)}=\sqrt{\frac{9.86}{2}\left(1+\frac{10}{5}\right)}=3.85 \mathrm{rad} / \mathrm{s}
$$

把 $\omega_{n}=3.85 \mathrm{rad} / \mathrm{s}$ 和 $\xi=0$ 分别代入式(7)、(8)、(10), 其中EI整形器中允许振荡百分比取通 值 $V_{t o l}=5 \%$, 由此得到吊车系统的ZV、ZVD、EI输入整形器:

$$
\begin{gathered}
Z V=\left[\begin{array}{cc}
A_{1} & A_{2} \\
t_{1} & t_{2}
\end{array}\right]=\left[\begin{array}{cc}
0.5 & 0.5 \\
0 & 0.82
\end{array}\right] \\
Z V D=\left[\begin{array}{ccc}
A_{1} & A_{2} & A_{3} \\
t_{1} & t_{2} & t_{3}
\end{array}\right]=\left[\begin{array}{ccc}
0.25 & 0.5 & 0.25 \\
0 & 0.82 & 1.64
\end{array}\right] \\
E I=\left[\begin{array}{ccc}
A_{1} & A_{2} & A_{3} \\
t_{1} & t_{2} & t_{3}
\end{array}\right]=\left[\begin{array}{ccc}
0.2625 & 0.4750 & 0.2625 \\
0 & 0.82 & 1.64
\end{array}\right]
\end{gathered}
$$

\section{4. 吊车运动系统动态特性仿真}

MATLAB软件中的Simulink工具箱可以用来对动态系统进行建模、仿真和分析, 在工业 和学术等领域得到广泛的应用。计算机仿真具有创建模型简单经济, 便于修改模型参数等优 点，从而大大减少设计周期，降低产品研发成本 ${ }^{[6]}$ 。

\section{1 吊车系统仿真模型的建立}

根据吊车运行系统传递函数, 在Simulink中建立吊车系统动态特性仿真框图 (图3)。仿真 系统的输入力为bang-bang力, 输出结果为负载在受力后产生的位移和相应摆角(图4)。

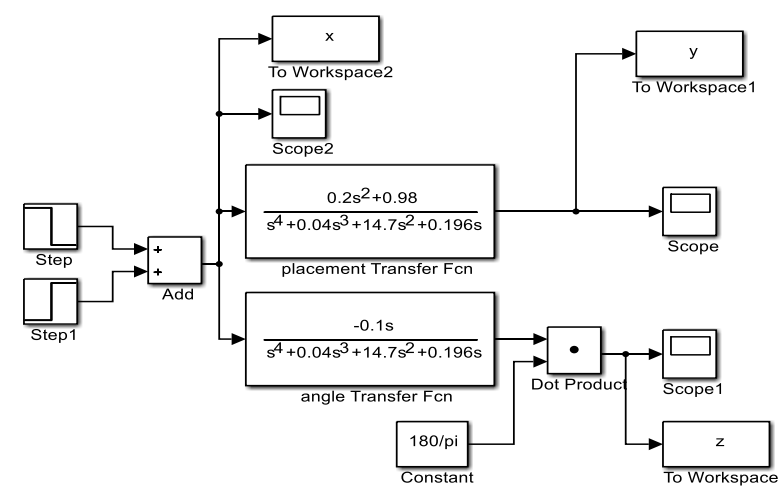

图3 吊车运行系统动态特性仿真框图

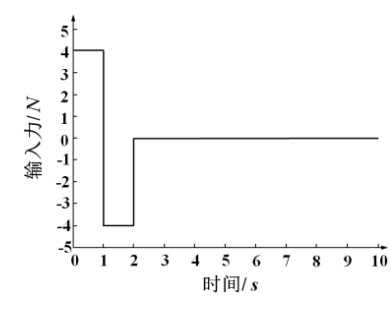

(a) bang-bang 输入力

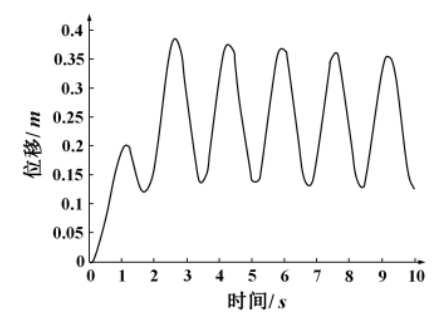

(b) 负载振荡位移曲线

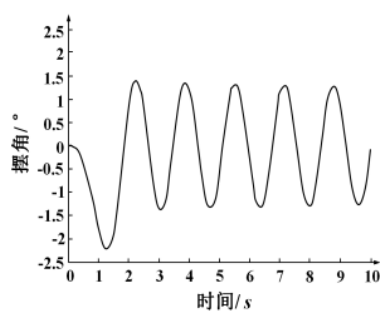

(c) 负载振荡摆角曲线

图4 吊车运行系统动态特性仿真结果 (未加整形器) 
系统仿真结果如图4所示，当在小车上施加输入力时，小车沿着轨道水平行走，小车在 $1 s$ 处, 驱动力从正的 $4 N$ 变为负的 $4 N$ （图 $4 \mathrm{a}$ ), 此时负载位移到达约 $0.2 m$ 位置处。输入力在 $2 s$ 时 刻变为 0 , 之后小车位移和角度的振荡取决于负载不停的做无阻尼摆动, 其中位移振幅约为 $0.12 m$ ，摆角幅值约为 $1.4^{\circ}$ 。

\section{2 基于输入整形的吊车运行系统仿真}

由于本文所建立的吊车系统输入参数一定, 且无外界不确定因素影响, 因此在对系统防 摇摆控制时, 优先选用 ZV整形器。ZV整形器由两个触发脉冲构成。由式(12)可以得到整形器 基于吊车自振频率和阻尼比的幅值和时间位置（表1）。

表1 ZV 整形器脉冲幅值与时间位置

\begin{tabular}{|c|c|c|}
\hline 脉冲 & 幅值 & 时间位置 \\
\hline 1 & 0.5 & 0 \\
\hline 2 & 0.5 & 0.82 \\
\hline
\end{tabular}

整形器与bang-bang输入力卷积得到系统的整形输入力(图5)。

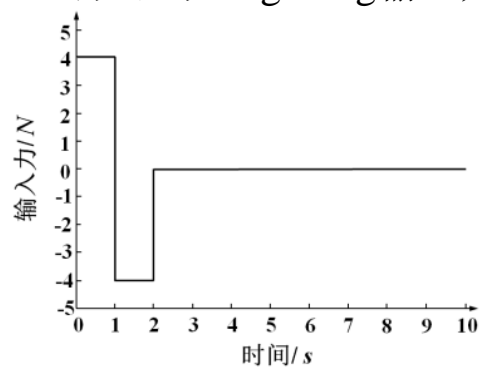

(a) 未整形bang-bang输入力

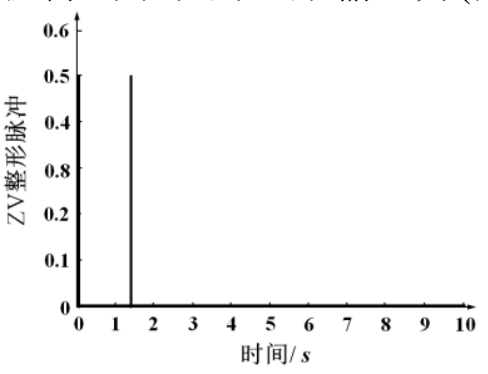

(b) 整形器

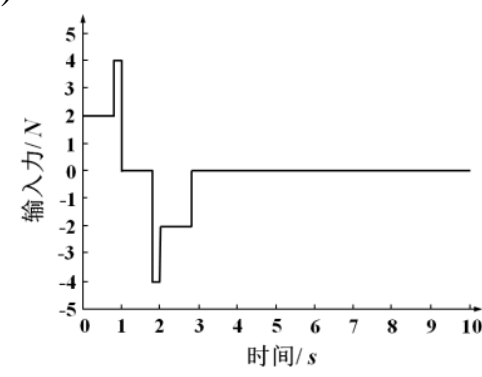

(c) 整形后输入力

图5 输入力与ZV整形器卷积仿真结果

将经过整形后得到的输入力输入到吊车系统模型中, 得到完整的基于输入整形的吊车系 统仿真框图(图6)。
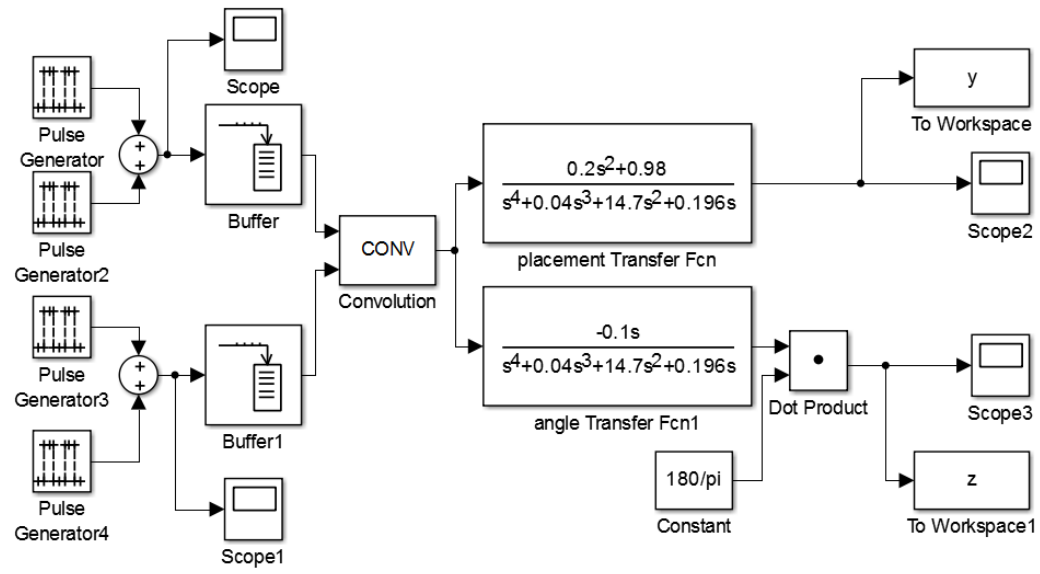

图6 基于输入整形的吊车运行系统仿真框图

仿真得到加入整形器后的负载摇摆位移和角度, 比较整形前后吊车系统仿真结果, 如图7 所示: 


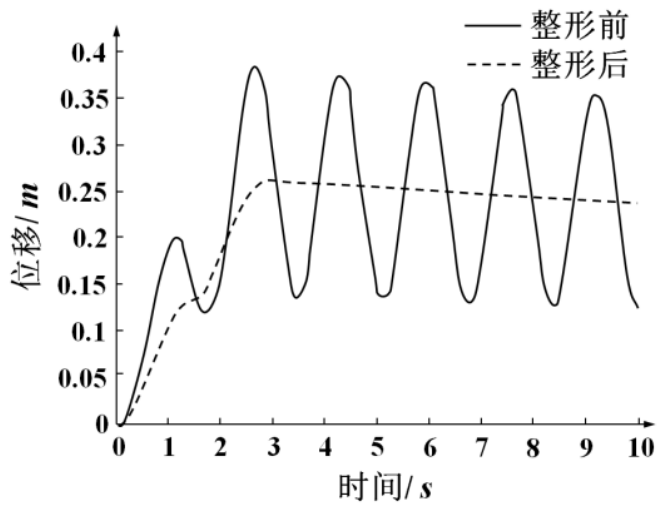

(a) 整形前后负载摇摆位移

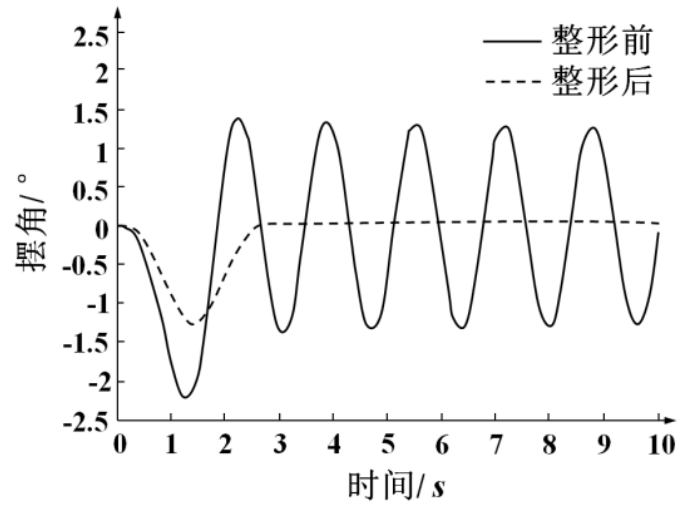

(b) 整形前后负载摇摆角度

图7 吊车运行系统动态特性仿真结果 (整形前后对比)

通过图形比较可以发现, 加了输入整形器后吊车摆动状况明显得到改善。当开始加输入 力时, 负载向负角度偏摆, 当返回到平衡位置处时便不再摆动(图7b)。同时比较负载摇摆位 移曲线可以发现, 加入整形器后的负载曲线要在时间上落后于整形前的负载摇摆曲线, 这表 明加了整形器后, 系统响应时间要变长。

\section{5. 结论}

本文对吊车运行系统进行了理论建模和仿真分析，利用Lagrange方程建立了吊车防摇摆 系统的数学模型, 严格推导出了系统的传递函数。提出基于输入整形技术抑制吊车负载摇摆 的方法, 对于三种整形器: 零振动 (ZV) 输入整形器、零振动微分 $(\mathrm{ZVD})$ 输入整形器和极不 灵敏 (EI) 输入整形器, 分析其各自特点, 并将该模型应用于吊车防摆问题上。通过仿真发 现输入整形技术能够有效抑制吊车运行情况下负载的摆动，验证了模型的正确性。

\section{References}

[1] ZHAO Zeng-xun. Research on Lagrange's Theory of Solving Algebraic Equations [D]. Xian : Northwest University. 2011.

[2] LIANG Yan-yang, XIONG Wei. Input Shaping Anti-swing Controller Design and Robustness Analysis for Crane System. Proceedings of the 31st Chinese Control Conference. 2012.

[3] HUANG Kai. Research on Intelligent Adaptive Anti-swing Control Method and Simulation for Crane [D]. Jiangsu : Nanjing Forestry University. 2007

[4] LI Wei. The Conditions and the Errors Abbreviating Model of Crane Load Sway [J]. Journal of Shandong Institute of ARCH and ENG. 1998, 13(3).

[5] MA Xi-Liang. Input Shaping Reduces the Residual Vibration of Little-friction System [D]. Guangxi : Guangxi University. 2008.

[6] GUO Li-Guan. Research and Simulation of Intelligent Anti-swing Control System for Port Container Crane [D]. Shanxi : Taiyuan University of Science \& Technology. 2009 